\title{
Short-baseline oscillation measurements at T2K
}

\author{
Javier Caravaca*t \\ IFAE University of Barcelona \\ E-mail: jcaravacalifae.es
}

The T2K experiment has searched for electron-neutrino disappearance in a electron-neutrino flux at short base-line due to oscillation to sterile neutrinos. The reactor and gallium anomalies, not explainable by the three neutrino framework and compatible with the hypothesis of a new mass eigenstate of $\sim 1 \mathrm{eV}^{2}$ are tested with the near detector (ND280) of T2K. At $280 \mathrm{~m}$ from the hadron production point and with an average electron-neutrino energy of $\sim 500 \mathrm{MeV}, \mathrm{ND} 280$ is sensitive to non-standard neutrino oscillations for a neutrino mass difference of $\sim 2 \mathrm{eV}^{2}$. The analysis of the electron-neutrino interaction rates as well as a good understanding of the backgrounds, allow to constrain the oscillation parameter space and to reject some regions of the gallium and reactor anomalies. On the other hand, the performances of the future nuPRISM detector on searches of electron-neutrino appearance at short base-line are reviewed.

16th International Workshop on Neutrino Factories and Future Neutrino Beam Facilities 25 -30 August, 2014

University of Glasgow, United Kingdom

\footnotetext{
*Speaker.

On behalf of the $\mathrm{T} 2 \mathrm{~K}$ collaboration
} 


\section{Introduction}

In the last two decades, several experiments have observed neutrino oscillations compatible with the hypothesis of neutrino mixing in a three active flavors basis, described by the PMNS matrix [1]. Nevertheless, there exist experimental data that cannot be accommodated in this framework: the deficit of $v_{e}$ originating from intense radioactive sources in the calibration of the solar neutrino gallium detectors SAGE [2, 3] and GALLEX [4] and $\bar{v}_{e}$ rates near nuclear reactors [5]. Those experiments cover $L / E$ values of order $1 \mathrm{~m} / \mathrm{MeV}$, where $L$ is the neutrino flight-path and $E$ is the neutrino energy, too large to observe any sizable effect for the standard neutrino mass differences. These anomalies can be interpreted as neutrino oscillations if the PMNS matrix is extended by introducing a new sterile neutrino $v_{s}(3+1$ model $)$ with a mass of order $1 \mathrm{eV}^{1}[5,6]$. The deficit would be due to $\stackrel{(-)}{v_{e}} \rightarrow v_{s}$ oscillations.

The T2K $v_{e}$ beam component is studied at ND280, the near detector of the T2K experiment [7], to search for $v_{e}$ disappearance. The analysis presented here considers $v_{e} \rightarrow v_{s}$ oscillations, given by the $v_{e}$ survival probability in the approximation of two neutrino mass states:

$$
P\left(v_{e} \rightarrow v_{e}\right)=1-\sin ^{2} 2 \theta_{\mathrm{ee}} \sin ^{2}\left(1.267 \frac{\Delta m_{\mathrm{eff}}^{2} L}{E}\right)
$$

where $\sin ^{2} 2 \theta_{\mathrm{ee}}$ is the oscillation amplitude, $\Delta m_{\mathrm{eff}}^{2}\left[\mathrm{eV}^{2}\right]$ is the mass squared difference between the new sterile mass state and the weighted average of the active standard mass states, with $L[\mathrm{~m}]$ and $E[\mathrm{MeV}]$.

With the given combination of $L$ and $E$, this analysis is sensitive to $v_{e}$ disappearance for $\Delta m_{\text {eff }}^{2} \gtrsim 2 \mathrm{eV}^{2}$ in a sample of $v_{e}$ charged current (CC) interactions [8]. A likelihood ratio fit to the reconstructed neutrino energy spectrum of the $v_{e} \mathrm{CC}$ interactions is used to test the sterile neutrino hypothesis. A high purity sample of photon conversions from $\pi^{0}$ decays is included in the fit to control the dominant background in the $v_{e}$ sample. In addition, a selection of $v_{\mu} \mathrm{CC}$ interactions at ND280 is used to constrain the neutrino flux and cross section uncertainties in order to substantially reduce the uncertainties on the predicted $v_{e} \mathrm{CC}$ interaction rate.

\section{2. $v_{e}$ flux of the $\mathbf{T} 2 \mathrm{~K}$ neutrino beam}

The T2K experiment [7] produces a neutrino beam that is studied at two different base-lines: ND280, at $280 \mathrm{~m}$ from the proton target, and SuperKamiokande, $295 \mathrm{~km}$ further. The current analysis is performed at the ND280 location, so we will omit any discussion on the far detector. The neutrino beam is composed mostly of $v_{\mu}$ with $8.8 \% \bar{v}_{\mu}, 1.1 \% v_{e}$ and $0.1 \% \bar{v}_{e}$ [12]. Due to ND280 sits $2.5^{\circ}$ from the primary proton beam direction (off-axis technique), the $v_{e}$ flux peaks at the neutrino energy of $\sim 500 \mathrm{MeV}$, as shown in Figure 1. The fluxes of $v_{e}$ and $\bar{v}_{e}$ are produced predominantly by $K^{ \pm}$and $K^{0}$ decays at high energies $(E>1 \mathrm{GeV})$, and mainly by $\mu$ decay in flight at low energies $(E<1 \mathrm{GeV})$ [12]. $K^{ \pm}$and $K^{0}$ tend to decay near the hadron production point due to

\footnotetext{
${ }^{1}$ Natural units adopted: $\mathrm{c}=1$
} 


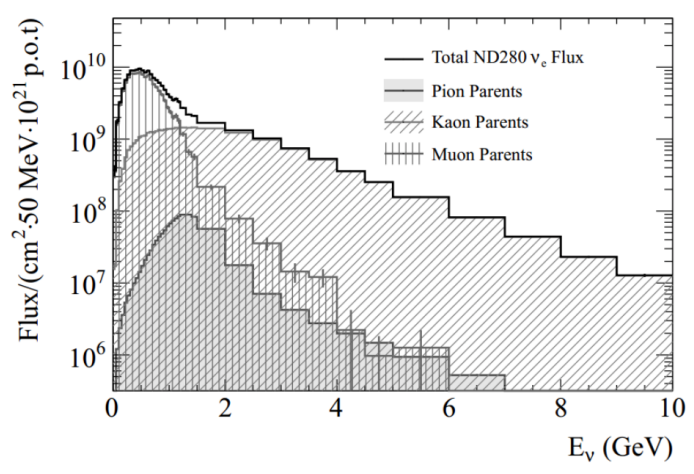

Figure 1: $v_{e}$ flux component predicted at ND280.

their short mean lifetime, while $\mu$ decay throughout the $96 \mathrm{~m}$ long decay volume, with a nearly flat decay length distribution. The average neutrino flight path, for $v_{e}$ selected in the analysis, is $244 \mathrm{~m}$.

\section{The ND280 detector}

ND280 is a magnetized series of detectors located $280 \mathrm{~m}$ from the T2K proton target at JPARC. The present analysis uses as a neutrino target, the two Fine Grained Detectors (FGDs [9]) made of polystyrene scintillator and filled with water layers, that corresponds to a total fiducial mass of about 1.6t. Three Time Projection Chambers (TPCs [10]) adjacent to the FGDs are used to identify the particle type and charge and measure the momentum. Electromagnetic calorimeters (ECal [11]), that surround the FGDs/TPCs (the Tracker) along the beam direction (Barrel ECal) and downstream (DsECal), additionally separate electron showers from muon tracks.

\section{4. $v_{e}$ and $\gamma$ event selections}

ND280 has collected neutrino interaction data for $5.9 \times 10^{20}$ protons on target. A sample of $v_{e}$ $\mathrm{CC}$ events is obtained by selecting electron-like events with the most energetic negatively charged track starting either in the FGD1 or FGD2 FV. Electron candidates are selected by combining the particle identification (PID) capabilities of the TPCs and ECals to reject $99.8 \%$ of muons, reaching an electron purity of $91.7 \%$ with a $\sim 5 \%$ component of misidentified muons. At this stage the dominant component $(\sim 65 \%)$ consist of electrons coming from photon conversions inside the FGD. Photons are generated inside or outside ND280 due to $\pi^{0}$ production in CC non quasi-elastic or neutral current interactions and this background needs to be further suppressed. In order to do that, vetoes that aim to eliminate this component are applied, achieving a background reduction of more than $50 \%$, being the photon conversion contamination of $29.3 \%$ in the final sample. 614 $v_{e}$ CC event candidates are selected with an efficiency of $26 \%$. Most of this background are due to interactions outside the fiducial volume (FV) that is not controlled by the $v_{\mu}$ cross-section and flux measurement and hence, suffer from bigger uncertainties. This stresses the need for a control sample to calibrate this important component and reach a precise measurement. 

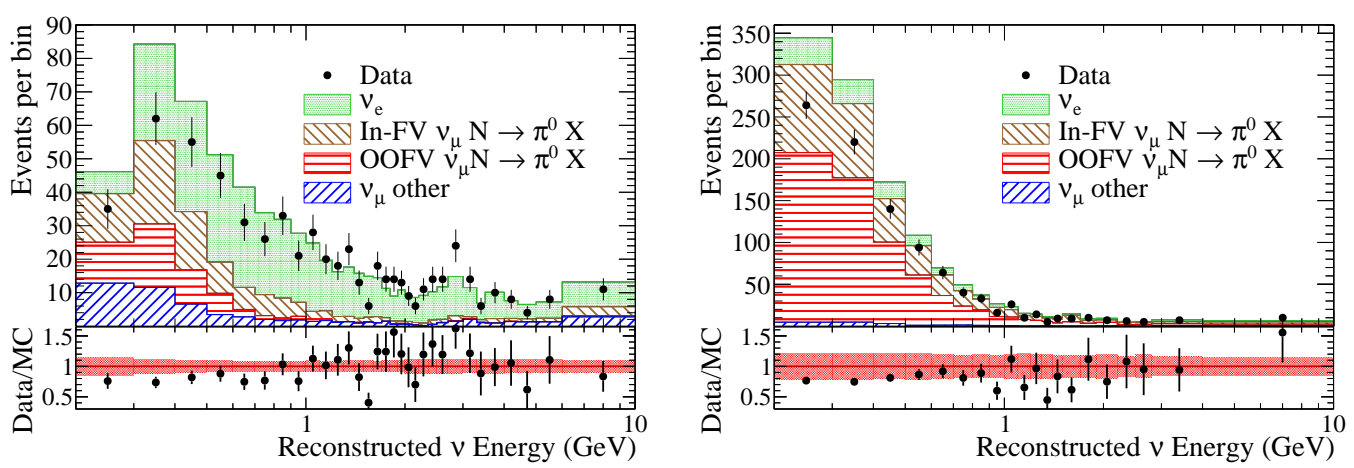

Figure 2: Reconstructed energy distributions of the $v_{e}$ (left) and control sample (right). The distributions are broken down by $v_{e}$ interactions (signal), photon background due to an interaction inside the FV (In-FV $v_{\mu} N \rightarrow \pi^{0} X$ ), outside the FV (OOFV $v_{\mu} N \rightarrow \pi^{0} X$ ) and all other sources of backgrounds ( $v_{\mu}$ other). The bottom part shows the ratios of the data to the expectation in the null oscillation hypothesis. The red bands correspond to the fractional systematic uncertainty while the black dots represent the data with only the statistical uncertainty.

\begin{tabular}{lccc}
\hline \hline Error source (\# param.) & $\begin{array}{c}v_{e} \text { sample } \\
(\text { sig+bkg) }\end{array}$ & $\begin{array}{c}v_{e} \text { sample } \\
(\text { sig only) }\end{array}$ & $\begin{array}{c}\text { control } \\
\text { sample }\end{array}$ \\
\hline$v_{\mu}-v_{e}$ common (40) & 4.4 & 5.2 & 6.7 \\
Unconstrained (5) & 3.7 & 3.0 & 17.8 \\
Detector + FSI (10) & 5.1 & 5.5 & 5.5 \\
\hline Total (55) & 7.6 & 8.1 & 19.9 \\
\hline \hline
\end{tabular}

Table 1: Fractional variation (RMS/mean in \%) of the expected total number of events for $v_{e}$ (all events and signal only) and control sample in the null oscillation hypothesis due to the effect of the systematic uncertainties. Correlations between systematics are taken into account.

A photon conversion control sample is selected by searching for near electron-like tracks with opposite charge and with an invariant mass smaller than $50 \mathrm{MeV}$. 989 events with a purity in photon events of $\sim 95 \%$ is reached, being the composition and kinematic similar from the photon background in the $v_{e}$ selection. A more detailed description of the selection of both the $v_{e}$ and the control sample is reported in [8].

The reconstructed $v_{e}$ energy spectrum $\left(E_{\text {reco }}\right)$, assuming a CCQE interaction, is inferred from the outgoing electron candidate momentum and angle. The behavior of the $E_{\text {reco }}$ shape is included in the analysis as the $v_{e}$ oscillation depends on the neutrino energy. Figure 2 shows the $E_{\text {reco }}$ distributions of the $v_{e}$ and the control samples.

Several sources of systematic errors are considered, namely, uncertainties on the flux, cross sections and detector response; and their effect on $E_{\text {reco }}$ are shown at the bottom of Figure 2 and summarized in Table 1. The large prior flux and cross-section uncertainties are reduced by a $v_{\mu}$ analysis at ND280 to the $4.4 \%$, which does not include the specific cross section systematics uncertainties of the $v_{e}$ analysis (like the ones induced by the out of FV events). 


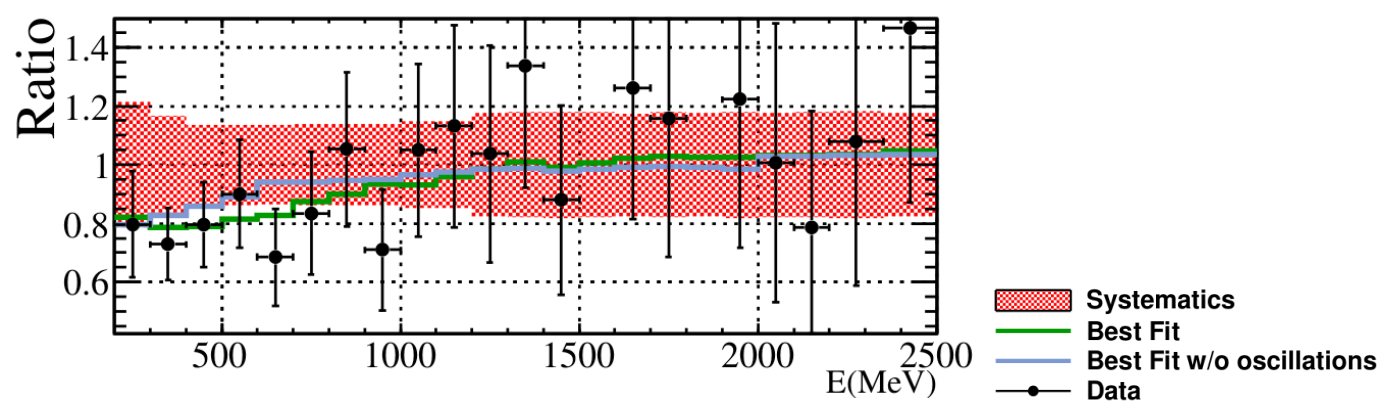

Figure 3: Subtraction of the $v_{e} E_{\text {reco }}$ distribution of the data and the nominal Monte-Carlo (dots). The subtraction between the Monte-Carlo after the fit and the nominal one is shown as well (green). Finally, the blue line shows the difference between the best fit Monte-Carlo without $v_{e}$ disappearance and the nominal one.

\section{Likelihood-fit and confidence intervals}

A binned log-likelihood fit is applied to the $E_{\text {reco }}$ distributions of the $v_{e}$ and photon samples in the region between $0.2 \mathrm{GeV}$ and $10 \mathrm{GeV}$. A so-called penalty term is added to the log-likelihood to include correlated Gaussian constraints of the systematic errors parameters. Those that drive the flux and most of the cross-sections are constrained by the $v_{\mu}$ analysis at ND280.

The oscillation parameters that best fit the current data are $\sin ^{2} 2 \theta_{\mathrm{ee}}=1.00$ and $\Delta m_{\mathrm{eff}}^{2}=$ $2.14 \mathrm{eV}^{2}$ and the post-fit agreement is shown in green in Figure 3. A good agreement is observed after the fit while a deficit at low energy was observed before the fit. The largest effect of the systematics is at low energy, as it is where we have most of the photon conversion background. Hence, in order to quantify this effect, the fit is performed for the null hypothesis, i.e. with the oscillation parameters fixed to zero, so that the possibility of $v_{e}$ disappearance is canceled. The result for this fit is showed by the blue line in Figure 3. In spite of that it fits the data rather well, including the $v_{e}$ disappearance turns to be in better agreement.

The p-value is calculated to quantify the compatibility of the ND280 with the null hypothesis within the systematic uncertainties and it is 0.061 , meaning that the null hypothesis is accepted at the $6.1 \%$ level. It is computed using toy MonteCarlos experiments built in the null hypothesis, as shown in Figure 4.

The bi-dimensional oscillation parameter space is studied to determine the confidence contours using the Feldman-Cousins method [13]. The 68\%, 90\% and 95\% confidence regions are shown in Figure 5. The exclusion region at $95 \% \mathrm{CL}$ is approximately given by $\sin ^{2} 2 \theta_{\mathrm{ee}}>0.3$ and $\Delta m_{\text {eff }}^{2}>7 \mathrm{eV}^{2}$. In Figure 5 the T2K excluded region at $95 \% \mathrm{CL}$ is compared with $v_{e}$ disappearance allowed regions from the gallium anomaly and reactor anomaly. The excluded regions from $v_{e}+{ }^{12} C \rightarrow{ }^{12} N+e^{-}$scattering data of KARMEN [14, 15] and LSND [16] experiments and solar neutrino and KamLAND data are also shown [17, 18, 19, 20, 21, 22, 23, 24, 25, 26, 27, 28, 29]. The $\mathrm{T} 2 \mathrm{~K}$ result excludes part of the gallium anomaly and a small part of the reactor anomaly allowed regions. 


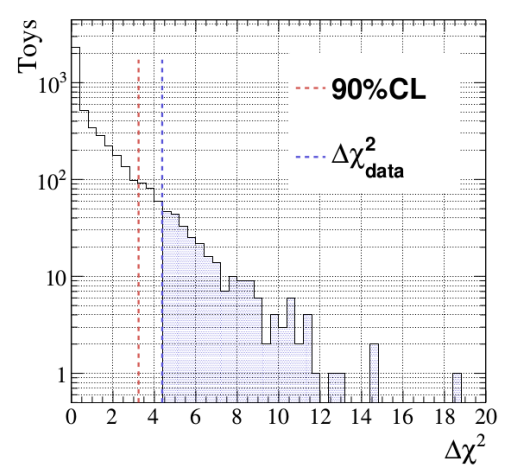

Figure 4: $\mathrm{p}$-value for the null hypothesis. The distribution corresponds to the $\chi^{2}$ values of 5000 toy MonteCarlos in the null hypothesis. They red dotted line correspond to the $90 \% \mathrm{CL} \chi^{2}$ critical value calculated using the toy experiments and the blue one corresponds to the $\chi^{2}$ value given by the fit on the real data.
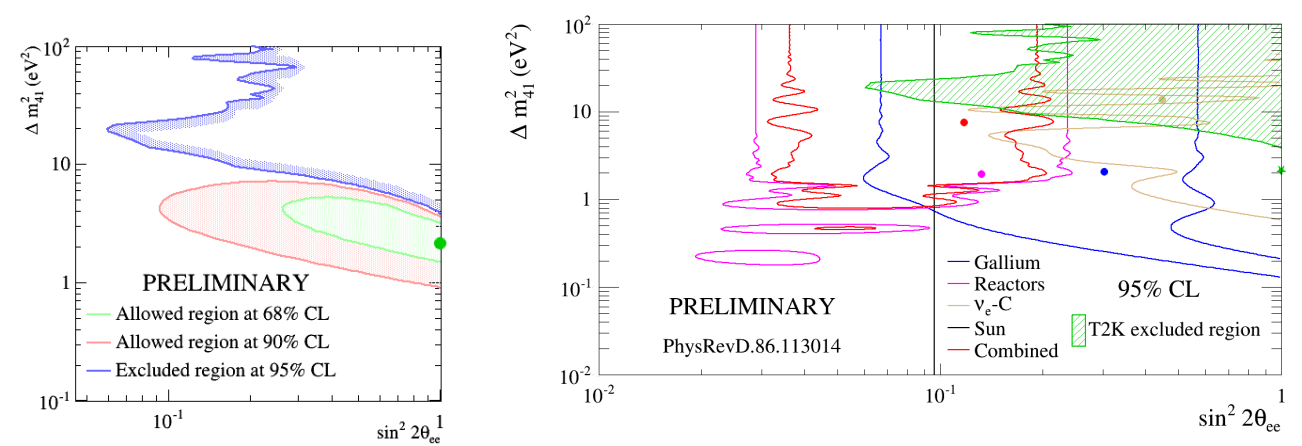

Figure 5: Confidence contours shown for ND280 at difference confidence levels (left) and for the 95\%CL for ND280 in comparison with the reactor and gallium anomalies, $v_{e}$ cross sections measurements in Carbon by KARMEN and LSND, the solar neutrino analysis and the combined fit of all the experiments except ND280 (right).

\section{Sensitivity of nuPRISM for $v_{e}$ appearance}

The nuPRISM idea is a new concept in the neutrino field. It consists of a long cylindric water Cerenkov detector located at a short distance from a beam neutrino source (typically $1 \mathrm{~km}$ ), being able to scan different off-axis angles and hence, different neutrino energy spectra [30]. This feature is specially interesting for sterile neutrinos as it opens the possibility of studying the anomalies as a function of the neutrino energy in an unique experiment. The current prediction is set for the JPARC neutrino beam with the a of $4 \mathrm{~m}$ inner radius located at $1 \mathrm{~km}$ distance from the proton target.

The sensitivity of such a detector to the appearance of $v_{e}$ in a $v_{\mu}$ beam in the minimal $3+1$ model sterile neutrino model has been calculated. The oscillation probability is given by the following expression:

$$
P_{e \mu} \equiv P\left(v_{\mu} \rightarrow v_{e}\right)=P\left(v_{e} \rightarrow v_{\mu}\right)=4\left|U_{e 4}\right|^{2}\left|U_{\mu 4}\right|^{2} \sin ^{2}\left(1.27 \Delta m_{\mathrm{eff}}^{2}\left[\mathrm{eV}^{2}\right] \frac{\mathrm{L}[\mathrm{m}]}{\mathrm{E}[\mathrm{MeV}]}\right)
$$




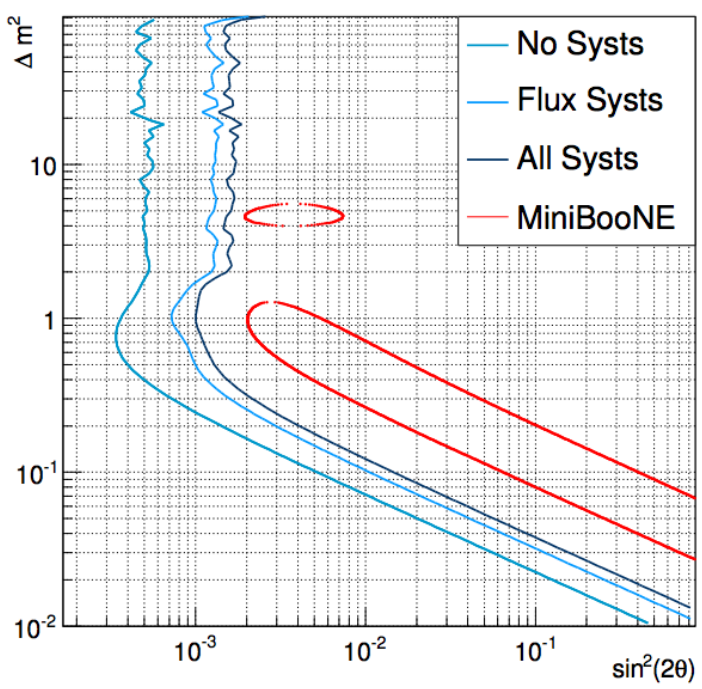

Figure 6: Sensitivity at the $90 \% \mathrm{CL}$ of nuPRISM for an exposure of $4.6 \times 10^{20}$ protons on target

For $4.6 \times 10^{20}$ proton on target (expected exposure after the $2018 \mathrm{~T} 2 \mathrm{~K}$ beam upgrade) the expected exclusion region for the 90\%CL covers the MiniBooNE anomaly [32] as shown in Figure 6. The analysis considers the flux and cross-section systematics in a very conservative way. No data driven constraints (from ND280) are utilized to decrease the size of the uncertainties and much more data is expected during the HyperKamiokande ([31]) era, so large room for future improvements exists. nuPRISM predictions are already very promising.

\section{Summary}

The ND280 detector is sensitive to neutrino mass differences of $\sim 2 \mathrm{eV}^{2}$ and the $v_{e}$ disappearance channel has been studied for an exposure of $5.9 \times 10^{20}$ protons on target. This is possible due to the development of a selection of $v_{e} \mathrm{CC}$ events with a good purity and the inclusion of the constraints for the flux and cross-section systematics provided by a $v_{\mu}$ analysis. A photon conversion control sample helps to reduce the low energy dominant systematic error. The p-value to the null hypothesis is 0.061 and the exclusion region at $95 \% \mathrm{CL}$ is approximately given by $\sin ^{2} 2 \theta_{\mathrm{ee}}>0.3$ and $\Delta m_{\mathrm{eff}}^{2}>7 \mathrm{eV}^{2}$.

Furthermore, the nuPRISM concept has been presented and the sensitivity to the $v_{e}$ appearance channel has been worked out. A very conservative analysis approach produces a $90 \%$ exclusion region that completely encloses the MiniBooNE anomaly.

\section{References}

[1] Z. Maki and M. Nakagawa and S. Sakata, Prog. Theor. Phys. 28, 870 (1962).

[2] J. N. Abdurashitov et al. (SAGE collaboration), Phys. Rev. C 59, 2246 (1999).

[3] J. N. Abdurashitov et al. (SAGE collaboration), Phys. Rev. C 73, 045805 (2006). 
[4] F. Kaether et al., Phys. Lett. B 685, 073006 (2010).

[5] G. Mention et al., Phys. Rev. D 83, 073006 (2011).

[6] M. A. Acero et al., Phys. Rev. D 78, 073009 (2008).

[7] K. Abe et al. (T2K Collaboration), Nucl. Instrum. Meth. A659, 106, (2011).

[8] K. Abe et al. (T2K Collaboration), Phys. Rev. D 89, 092003 (2014).

[9] P. Amaudruz et al., Nucl. Instrum. Methods A696, 1 (2012).

[10] N. Abgrall et al., Nucl. Instrum. Methods A637, 25 (2011).

[11] D. Allan, et al., JINST, 8, P10019 (2013).

[12] K. Abe et al. (T2K Collaboration), Phys. Rev. D 87, 012001 (2013).

[13] G. J. Feldman and R. D. Cousins, Phys. Rev. D 57, 3873 (1998).

[14] B.E. Bodmann et al. (KARMEN Collaboration), Phys. Lett. B 332, 251 (1994).

[15] B. Armbruster et al., Phys. Rev. C 57, 3414 (1998).

[16] L. B. Auerbach et al. (LSND Collaboration), Phys. Rev. C 64, 065501 (2001).

[17] F. Kaether, W. Hampel, G. Heusser, J. Kiko, and T. Kirsten, Phys. Lett. B 685, 47 (2010).

[18] B. T. Cleveland et al. (Homestake Collaboration), Astrophys. J. 496, 505 (1998).

[19] J.N. Abdurashitov et al. (SAGE Collaboration), J. Exp. Theor. Phys. 95, 181 (2002).

[20] J. Hosaka et al. (Super-Kamkiokande Collaboration), Phys. Rev. D 73, 112001 (2006).

[21] J. Cravens et al. (Super-Kamkiokande Collaboration), Phys. Rev. D 78, 032002 (2008).

[22] K. Abe et al. (Super-Kamkiokande Collaboration), Phys.Rev. D 83, 052010 (2011).

[23] M. Smy (Super-Kamiokande Collaboration), in XXV International Conference on Neutrino Physics and Astrophysics, 2012 (Neutrino, Kyoto, 2012).

[24] Q. R. Ahmad et al. (SNO Collaboration), Phys. Rev. Lett. 89, 011302 (2002).

[25] B. Aharmim et al. (SNO Collaboration), Phys. Rev. C 72, 055502 (2005).

[26] B. Aharmim et al. (SNO Collaboration), Phys. Rev. Lett. 101, 111301 (2008).

[27] G. Bellini et al. (Borexino Collaboration), Phys. Rev. Lett. 107, 141302 (2011).

[28] G. Bellini et al. (Borexino Collaboration), Phys. Rev. Lett. 108, 051302 (2012).

[29] A. Gando et al. (KamLAND Collaboration), Phys. Rev. D 83, 052002 (2011).

[30] S. Bhadra et al. (nuPRISM Collaboration), http://arxiv.org/abs/1412.3086 (2014).

[31] K. Abe et al. (HyperKamiokande Collaboration), http://arxiv.org/abs/1109.3262 (2011).

[32] A.A. Aguilar-Arevalo et al. (MiniBooNE Collaboration), Phys.Rev.Lett. 110161801 (2013). 